\title{
AVE - Method for 3D Visualization of Search Results
}

\author{
Wojciech Wiza, Krzysztof Walczak, and Wojciech Cellary \\ Department of Information Technologies, Poznan University of Economics \\ Mansfelda 4, 60-854 Poznan, Poland \\ \{wiza,walczak, cellary\}@kti.ae.poznan.pl
}

\begin{abstract}
A novel approach to three-dimensional visualization of search results is presented. In the proposed method a search result is progressively visualized by a sequence of customizable $3 \mathrm{D}$ interfaces. The interface applied at each visualization stage is selected with regards to properties of the search result and user interactions. A user may also change mapping of search result properties to visual attributes of the interfaces.
\end{abstract}

\section{Introduction}

With the creation of the World Wide Web service, which caused the Internet to grow exponentially, the question: "IS the information accessible" is often replaced by: "WHERE the information can be found". The answer to this question comes with search engines - specialized systems that store data about WHAT and WHERE information is kept.

To provide better search capabilities, numerous research initiatives focus on either invention of new technologies for Internet search (like P2P systems) or improving existing search systems. While the first solution brings many uncertainties and is in general difficult to implement, the later can be realized on many different stages of information indexing and retrieval. Examples include use of more and more sophisticated crawlers, improvements in data retrieval by the use of new indexing techniques and query languages, and new methods of information visualization. The later seems to be a little neglected, even though it is a promising method of enhancing search systems.

A natural way to enhance visualization capabilities of search engines is the use of graphical interfaces. In the recent years there were several attempts to create graphical search interfaces. Many of them used two-dimensional graphics (e.g. Antarctica [1], InXight [2]), but there were also several attempts to apply three-dimensional visualization, e.g. 3D cards [3], Antarctica 3D, VR-VIBE [4][5], Cat-a-Cone [6], Nirve [7], or ViOS system [8]. The tendency to use 3D visualization techniques increases with the advances in 3D technology. Current standardization efforts in this field and availability of cheap and powerful 3D graphic accelerators allow to anticipate wider use of 3D interfaces in the future.

Most of the 3D visualization interfaces proposed up to now share similar drawbacks: data visualization is performed in a single universal 3D environment, which in many cases causes improper presentation of information and thus decreases user perception; lack of presentation of aggregated data first, and then - in response to user interaction - more specific data; the interfaces either require installation of dedicated software or have limited interaction capabilities due to the use of purely standardbased 3D browsers. 


\section{The AVE Method}

To solve the above problems a new approach to 3D visualization of search results, called AVE (Adaptive Visualization Environments), has been developed. The fundamental concept of the AVE method is the creation of an interface that permits visualization of the entire search result. Such an interface presents a global view of retrieved search result instead of the first dozens of most relevant documents. Data presented using the AVE method are not divided into a sequence of chunks or pages. This permits a user not only to browse through the information but also to understand its nature. Appropriately constructed and applied interface permits a user to perceive trends in data faster and with bigger precision through differences in color, shape, connectedness, continuity, symmetry, etc. Use of 3D environments enhances the number of available visualization dimensions in a natural way and gives a user a multi-criterion overview of the retrieved data. A 3D environment permits a user to change a viewpoint within the scene, therefore allows to exploit the entire data set from the best perspective.

In the AVE method, depending on the search result volume, the visualization system may present data in detailed view or classify data using one or more classification criteria (cf. Fig. 1). The classification criteria may be selected automatically by the system based on search result properties, can be pre-selected by a user, or both.

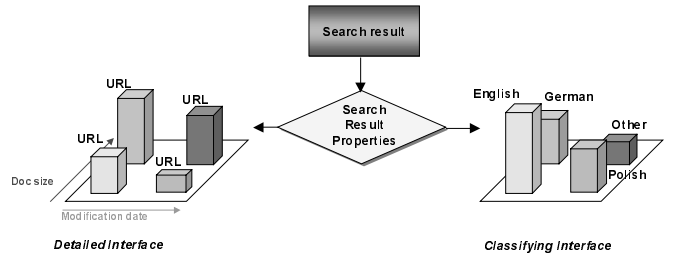

Fig. 1. Selection of the interface based on search result properties

While the search results may differ significantly in the number of documents and other properties (e.g., number of domains, hosts, document types), it is impossible to find a single universal interface that would reflect all possible search results in a readable way. Therefore, in the proposed AVE method, a number of automatically or manually selected interfaces are used. The interfaces differ in their visualization capabilities. Selection of the best-fitted interface may be performed automatically by the visualization system, manually by a user, or both methods may be combined. An automatic selection of the interface is based on properties of the search result, like number of retrieved URLs, number of languages and semantic relationships between documents. In the manual mode a user may select an interface, which in his/her opinion shows the result in the most appropriate way. Therefore, main factors, which influence manual selection of the interface are user preferences and experience. Joining these two methods of interface selection, a user may choose an interface from a set of interfaces pre-selected by a system, or the system may choose the best environment from the list of interfaces selected by the user.

A user explores the search result in a number of subsequent steps (cf. Fig. 2). Using different levels of abstraction and applying the most appropriate 3D environment at each step, the proposed method permits to navigate from a classified, aggregated 
view of the entire search result, through aggregated views of sub-results, up to precise visualization of particular documents of user interest.

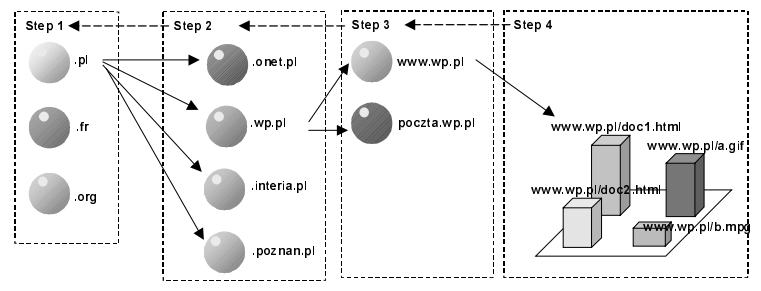

Fig. 2. Exploration of the search result from general to detailed view

In the proposed method, a user may apply a number of different environments to the same search result. This permits to visualize retrieved data in different ways to better understand the information displayed and to detect trends and patterns.

Another technique that improves perception of the visualized data in the AVE method, is flexible assignment of search result properties to visual attributes of the interfaces (cf. Fig. 3). A user may decide which characteristic of the retrieved data should be visualized by a particular dimension in the $3 \mathrm{D}$ environment, e.g. the size of a document can be reflected in the size of an object. Possible mapping choices are described by mapping functions defined in the interface specification. These functions guarantee proper transformation from domains of search result properties to domains of visual attributes.

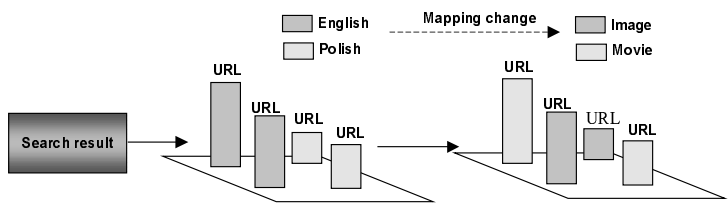

Fig. 3. Selection of the mapping for search result attributes

The AVE method is independent of the search engine database, therefore it can be applied to virtually every commercial (like Altavista or Google) or non-commercial search engine. For testing purposes a search system, implementing the proposed method, has been built. This system, called Periscope [9], uses a simple database (loaded with data gathered by a web crawler) and a set of interfaces (cf. Fig. 4). More information about the interfaces in Periscope system can be found in [10].

\section{Conclusions}

First trials with end-users show that the AVE method implemented in the Periscope system can be efficiently used for Web searching. Although, the system response time is sometimes higher than in case of popular search engines (like Altavista or Google) reaching up to 15 seconds for complex queries, end-users felt that the accuracy of the information retrieved was higher, especially in the case of vague initial information requirements. Current tests focus on the ergonomics and user perception of different 
3D interfaces. Future works include improvement of interface models based on results of system evaluation performed by end-users.
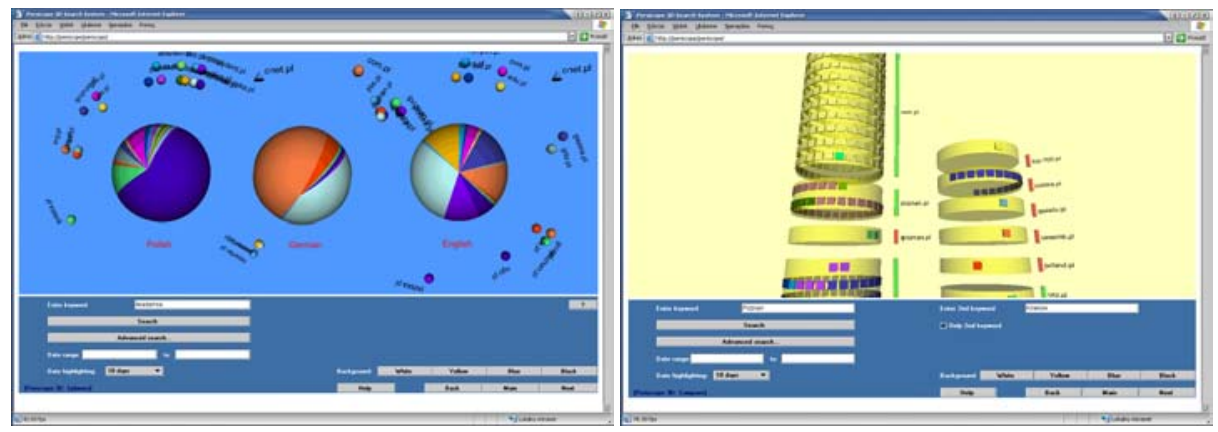

Fig. 4. Example interfaces used in the Periscope system

\section{References}

1. Antarctica homepage: http://www.antarcti.ca/

2. InXight homepage: http://www.inxight.com/map/

3. Mukherjea, S., Y. Hara: Visualizing World-Wide Web Search Engine Results, ICIV International Conference, July 14-16, 1999 London, England

4. Benford, B., D. Snowdon, C. Greenhalgh, R. Ingram, I. Knox, C. Brown: VR-VIBE: A Virtual Environment for Co-operative Information Retrieval, Eurographics'95, 30th August - 1st September, Maastricht, The Netherlands, pp 349-360.

5. VR-VIBE homepage: http://www.emptiness.org/vr/vrvibe.html

6. Cat-A-Cone project homepage: http://www.sims.berkeley.edu/ hearst/cac-overview.html

7. NIRVE project homepage: http://www.itl.nist.gov/iaui/vvrg/cugini/uicd/nirve-home.html

8. ViOS - how does it works?: http://www.howstuffworks.com/vios.htm

9. Periscope project homepage: http://periscope.kti.ae.poznan.pl/periscope/

10. Wiza, W., K. Walczak, W. Cellary: Adaptive 3D Interfaces for Search Result Visualization IADIS International Conference e-Society, 3-6 June 2003, Lisbon, Portugal 